\title{
TMJ Inflammation Increases Fos Expression in the Nucleus Raphe Magnus Induced by Subsequent Formalin Injection of the Masseter or Hindpaw of Rats
}

\author{
By \\ Sang-Hoon OH, Hiroki IMBE and Yasutomo IWAI-LIAO \\ Department of Oral Anatomy, Osaka Dental University, Kuzuhahanazono-cho 8-1, Hirakata-shi, 573-1121, Japan \\ - Received for Publication, April 28, $2006-$ \\ Key Words: Descending system, Fos, Inflammation, Temporomandibular joint, Rat

\begin{abstract}
Summary: The study was designed to examine the effect of persistent temporomandibular joint (TMJ) inflammation on neuronal activation in the descending pain modulatory system in response to noxious stimulus. Formalin was injected into the left masseter muscle or hindpaw of rats 10 days after injection of the left TMJ with saline or complete Freund's adjuvant (CFA). The results showed that 10-day persistent TMJ inflammation (induced by CFA) alone did not induce a significant increase in Fos-like immunoreactive (Fos-LI) neurons in the rostral ventromedial medulla (RVM) or locus coeruleus (LC), but that formalin injection of the masseter muscle or hindpaw induced a significant increase in Fos-LI neurons in the RVM and LC of rats with and without TMJ inflammation $(P<0.05)$. However, persistent TMJ inflammation significantly increased Fos-LI neurons in the nucleus raphe magnus (NRM) induced by subsequent formalin injection of the masseter muscle and hindpaw $(\mathbf{7 0 . 2} \%$ increase and $53.8 \%$ increase, respectively, over the control TMJsaline-injected rats; $P<\mathbf{0 . 0 5}$ ). The results suggest that persistent $T M J$ inflammation increases neuronal activity, in particularly in the NRM, by the plastic change of the descending pain modulatory system after ipsilateral application of a noxious stimulus to either orofacial area or a spatially remote body area.
\end{abstract}

Temporomandibular disorders (TMDs) cause various types of pain involving the masticatory muscles and temporomandibular joint ${ }^{1)}$. Clinical studies have described TMD patients having generalized hyperalgesia beyond the orofacial and neck regions ${ }^{2-4)}$. Not only a significantly lower pain threshold in the masticatory muscles has been found in TMD patients than in healthy persons, but dysfunction in the endogenous pain control system as well ${ }^{5,6}$. A recent study has reported that TMD induces widespread neural changes in supraspinal structures involved in the central nociceptive pathways $^{7)}$.

Inflammation of the TMJ and masticatory muscles is likely to contribute to the induction and/or maintenance of persistent TMD pain ${ }^{8,9}$. In earlier studies the authors injected complete Freund's adjuvant (CFA) into the rat TMJ and observed thermal and mechanical hyperalgesia of the facial skin ipsilateral to the inflamed TMJ that persisted for 2 weeks, and enhancement of orofacial nocifensive behavioural activity induced by subsequent nox- ious stimulus was observed in the same TMJ inflammation model ${ }^{10-13)}$. There have been several studies on persistent TMJ inflammation, and they have shown an increase in expression of Fos, a protein product of the c-fos immediate early gene (IEG), and opioid gene accompanied by neuronal hyperexcitability in the spinal trigeminal nucleus (Vsp) ${ }^{10,11,14-16)}$. Thus, the results of these studies have suggested that central neuronal changes play a critical role in the induction and maintenance of persistent orofacial pain in TMJ inflammation.

A study on chronic TMJ monoarthritis of rats described a subsequent mustard oil injection of the TMJ contralateral to inflammation led to increased excitability of trigeminal brainstem neurons ${ }^{17)}$. Another study reported that nocifensive behaviours evoked by formalin injection of the hindpaw of rats were exaggerated by chronic TMJ inflammation ${ }^{18)}$. Although the studies indicated that the facilitation of nociceptive responses in spatially remote body areas by chronic TMJ inflammation could be explained by a mechanism of sensitization

Correspondence to: Hiroki Imbe, D.D.S., Ph.D., Dept. of Oral Anatomy, Osaka Dental University, Kuzuhahanazono-cho 8-1, Hirakata City, 573-1121, Japan. E-mail: imika@js9.so-net.ne.jp 
of supraspinal structures in the descending pain modulatory system, how the inflammation affects modulatory neuronal activity following a noxious stimulus was not elucidated.

The rostral ventromedial medulla (RVM), which contains the nucleus raphe magnus (NRM) and nucleus reticularis gigantocellularis pars alpha (GiA), and the locus coeruleus (LC) have been found to play crucial roles in the descending pain modulatory system ${ }^{19-21)}$. Moreover, one study indicated that electrical or chemical stimulation of the RVM arouses biphasic (facilitatory and inhibitory) modulation of spinal nociceptive transmission ${ }^{22)}$. Since the RVM has been demonstrated to contribute to the maintenance of inflammatory pain and neuropathic pain, a great deal of attention has been focused on the RVM ${ }^{23,24)}$.

Fos expression excited by various stimuli has been widely used to assess neuronal activation, and is known to contribute to the establishment of longterm functional changes in the central nervous system $^{25,26)}$. Some previous studies have presented histological evidence that formalin injection of the hindpaw or face of rats induces Fos expression in the RVM and $\mathrm{LC}^{27,28)}$. Furthermore, Fos expression in NRM serotonergic neurons was assumed to activate the descending pathways involved in behavioural hyperalgesia ${ }^{29)}$.

In the present study we investigated the effects of persistent TMJ inflammation on Fos expression in supraspinal structures evoked by formalin injection of the masseter muscle or hindpaw, in order to study plastic neuronal changes in the descending pain modulatory system in TMD patients.

\section{Materials and Methods}

\section{Animal preparation}

Male Sprague-Dawley rats (150-250 g body weight; Japan SLC, Shizuoka, Japan) were used in this study. The protocol (No. 06-02016: Identification of the mechanism of secondary hyperalgesia in TMJ chronic inflammation model) was approved by the Ethics Review Board of Osaka Dental University and implemented in accordance with the ethical guidelines for the treatment of animals of the International Association for the Study of Pain ${ }^{30)}$.

\section{TMJ inflammation}

Complete Freund's adjuvant (Mycobacterium tuberculosis, Calbiochem, CA, USA) suspended in an oil:saline $(1: 1)$ emulsion was used as the inflammatory agent. Orofacial thermal hyperalgesia and mechanical allodynia, peaking at 1 day and persisting for 2 weeks after CFA injection into the rat TMJ, were observed as described in previous studies $^{10,11)}$. Histologically, acute inflammation occurred in soft tissues around the TMJ at 6 hours after the CFA injection and shifted to a chronic inflammation phase at day $10^{31)}$. We also observed that Fos expression in the Vsp peaked between day 1 and day 3 and gradually recovered by day 10 after the CFA injection, as reported in a previous study ${ }^{15)}$. The increased nocifensive effects evoked by formalin injection of the masseter muscle and hindpaw were observed at 7-10 days after CFA injection $12,13,18,32$ ).

\section{Experimental groups}

Rats were divided into the following 6 groups (Fig. 1):

1) A TMJ-saline group $(n=4)$, anesthetized with sodium pentobarbital $(50 \mathrm{mg} / \mathrm{kg}$, i.p.) and in which the left TMJ was injected with saline (0.05 mL) 10 days before sacrifice.

2) A TMJ-CFA group $(\mathrm{n}=4)$, anesthetized with sodium pentobarbital $(50 \mathrm{mg} / \mathrm{kg}$, i.p.) and in which the left TMJ was injected with CFA (0.05 mL, $0.025 \mathrm{mg}) 10$ days before sacrifice.

3) A TMJ-saline + Masseter-formalin group $(n=4)$, in which the mid-region of the left masseter muscle was injected with $5 \%$ formalin $(0.05 \mathrm{~mL}) 10$ days after injection of the left TMJ with saline.

4) A TMJ-CFA + Masseter-formalin group $(\mathrm{n}=5)$, in which the left masseter muscle was injected with formalin 10 days after injection of the left TMJ with CFA.

5) A TMJ-saline + Hindpaw-formalin group $(\mathrm{n}=4)$, in which the left hindpaw was injected with formalin 10 days after saline injection of the left TMJ.

6) A TMJ-CFA + Hindpaw-formalin group $(\mathrm{n}=4)$, in which the left hindpaw was injected with formalin 10 days after CFA injection of the left TMJ.

\section{Tissue preparation and immunohistochemical analysis}

The rats were euthanized with $\mathrm{CO}_{2}$ gas $2 \mathrm{~h}$ after formalin injection of the masseter or hindpaw and 10 days after the saline or CFA injection. They were then transcardially perfused with $100 \mathrm{~mL}$ of saline solution followed by $500 \mathrm{~mL}$ of ice-cold $4 \%$ paraformaldehyde in $0.1 \mathrm{M}$ phosphate buffer (PB) at $\mathrm{pH}$ 7.4. The brainstems were removed, placed in the same fixative overnight at $4^{\circ} \mathrm{C}$, and then transferred to $30 \%$ sucrose $(\mathrm{w} / \mathrm{v})$ in $0.1 \mathrm{M} \mathrm{PB}$ for 3 days for tissue cryoprotection. The brainstem were cut into $30 \mu \mathrm{m}$ sections at $-20^{\circ} \mathrm{C}$ with a cryostat, and then serially transferred to multi-well tissue culture 


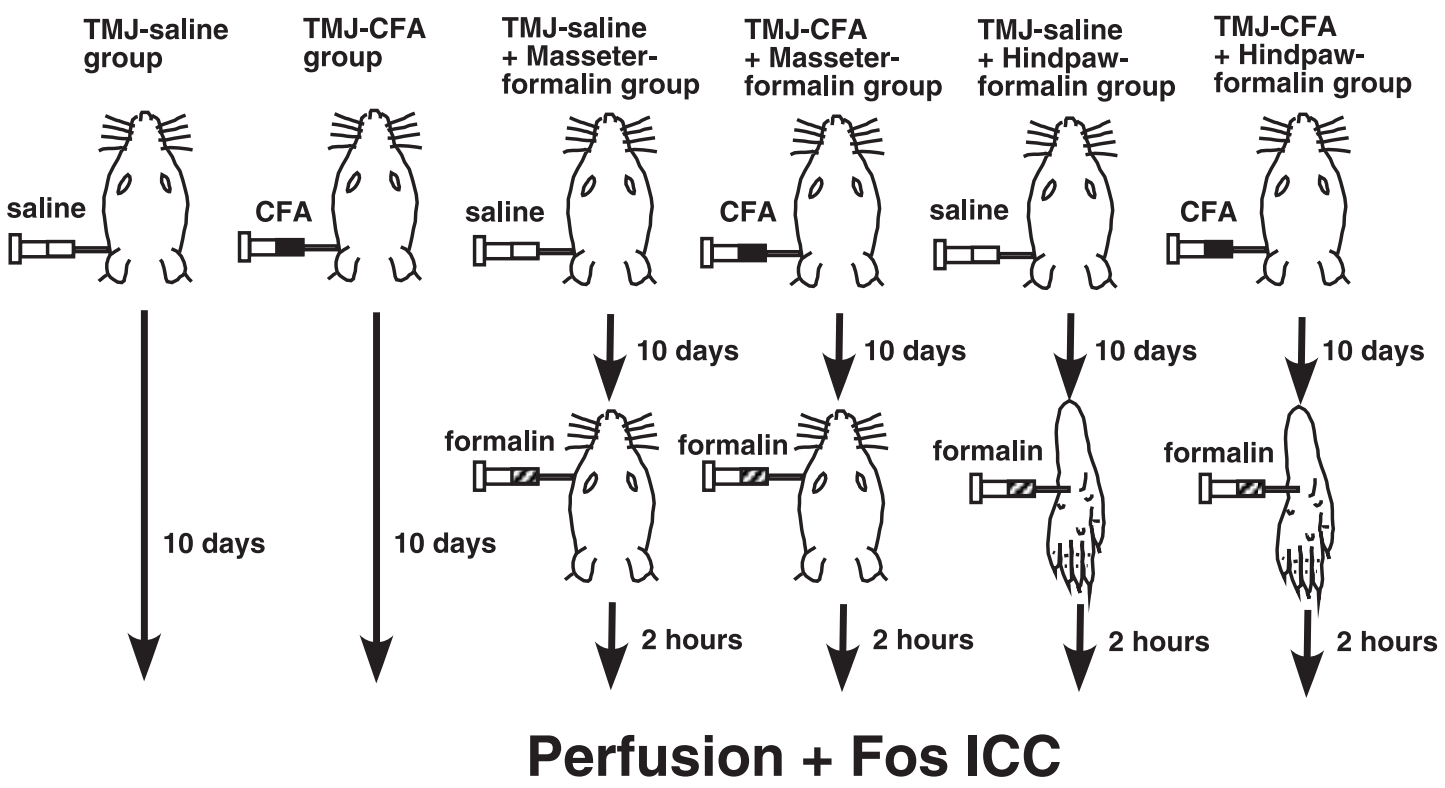

Fig. 1. Experimental design of the TMJ-saline, TMJ-CFA, TMJ-saline + Masseter-formalin, TMJ-CFA + Masseter-formalin, TMJsaline + Hindpaw-formalin and TMJ-CFA + Hindpaw-formalin groups. Saline or CFA was injected into the TMJ 10 days prior to formalin injection $(5 \%, 0.05 \mathrm{~mL})$ of the masseter muscle or hindpaw.

plates containing 0.1 M Tris-buffered saline (TBS). Every sixth section (between $-8.96 \mathrm{~mm}$ and -11.30 $\mathrm{mm}$ from the bregma) of each brainstem was collected and processed for immunohistochemical study and analysis.

Free-floating sections were washed for $30 \mathrm{~min}$ with TBS containing $0.1 \%$ Triton X-100 (TBST), and after incubation in $1 \%$ hydrogen peroxide in TBST for $30 \mathrm{~min}$ followed by another three washes with TBST, they were incubated for $1 \mathrm{~h}$ at room temperature (RT) in TBST containing 3\% bovine serum albumin (BSA). The sections were incubated with the primary antibody (rabbit polyclonal antibody against c-Fos, Ab-5, dilution 1:20000, Oncogene Research products, CA, USA) in TBST with $3 \%$ BSA for $24 \mathrm{~h}$ at $4{ }^{\circ} \mathrm{C}$. After incubation, sections were washed again in TBST and then incubated for $1 \mathrm{~h}$ at RT with the biotinylated secondary antibody (goat anti-rabbit, Vector Laboratories, CA, USA). The sections were then washed twice in TBST, incubated with avidin-biotin-peroxidase complex (Elite ABC kit, Vector Laboratories) for $1 \mathrm{~h}$ at RT, and washed again in TBST. The horseradish peroxidase reaction was developed in TBS ( $\mathrm{pH} 7.5)$ containing $0.05 \% \quad 3,3^{\prime}$-diaminobenzidine tetrahydrochloride, $0.2 \%$ nickel sulfate and $0.01 \%$ hydrogen peroxide. The control sections processed without primary antibody incubation were not stained. The brainstem sections were mounted on slides, air-dried, dehydrated in a graded ethanol series, cleared in xylene, and cover-slipped.
The number of Fos-like immunoreactive (FosLI) neurons in the nucleus raphe magnus (NRM), nucleus reticularis gigantocellularis pars alpha (GiA), ventral nucleus reticularis gigantocellularis (Gi), and nucleus raphe pallidus (NRP) in $10 \mathrm{sec}-$ tions (between $-9.68 \mathrm{~mm}$ and $-11.30 \mathrm{~mm}$ from the bregma) of each brainstem was counted under a light microscope. Fos-LI neurons in the locus coeruleus (LC) in 10 sections (between $-8.96 \mathrm{~mm}$ and $-10.58 \mathrm{~mm}$ from the bregma) of each brainstem were also counted. Nuclear and laminar boundaries were defined according to a cytoarchitectonic atlas and other previous studies ${ }^{33,34)}$. The total number of Fos-LI neurons within the designated rostrocaudal levels (by means \pm S.E.M.) in the brainstem nuclei of each experimental group were statistically analyzed by ANOVA with Fisher's PLSD for inter-group comparisons $(p<0.05)$.

\section{Results}

\section{Fos-LI expression}

We observed very few Fos-LI neurons in the RVM or LC at day 10 after injection of the TMJ with saline or CFA (Fig. 2: B, G and L; Fig. 2: C, H and $\mathrm{M}$ ). Formalin injection of the masseter muscle in both the presence and absence of chronic TMJ inflammation induced an increase in Fos-LI neurons, particularly in the NRM of the RVM (Fig. 2: 

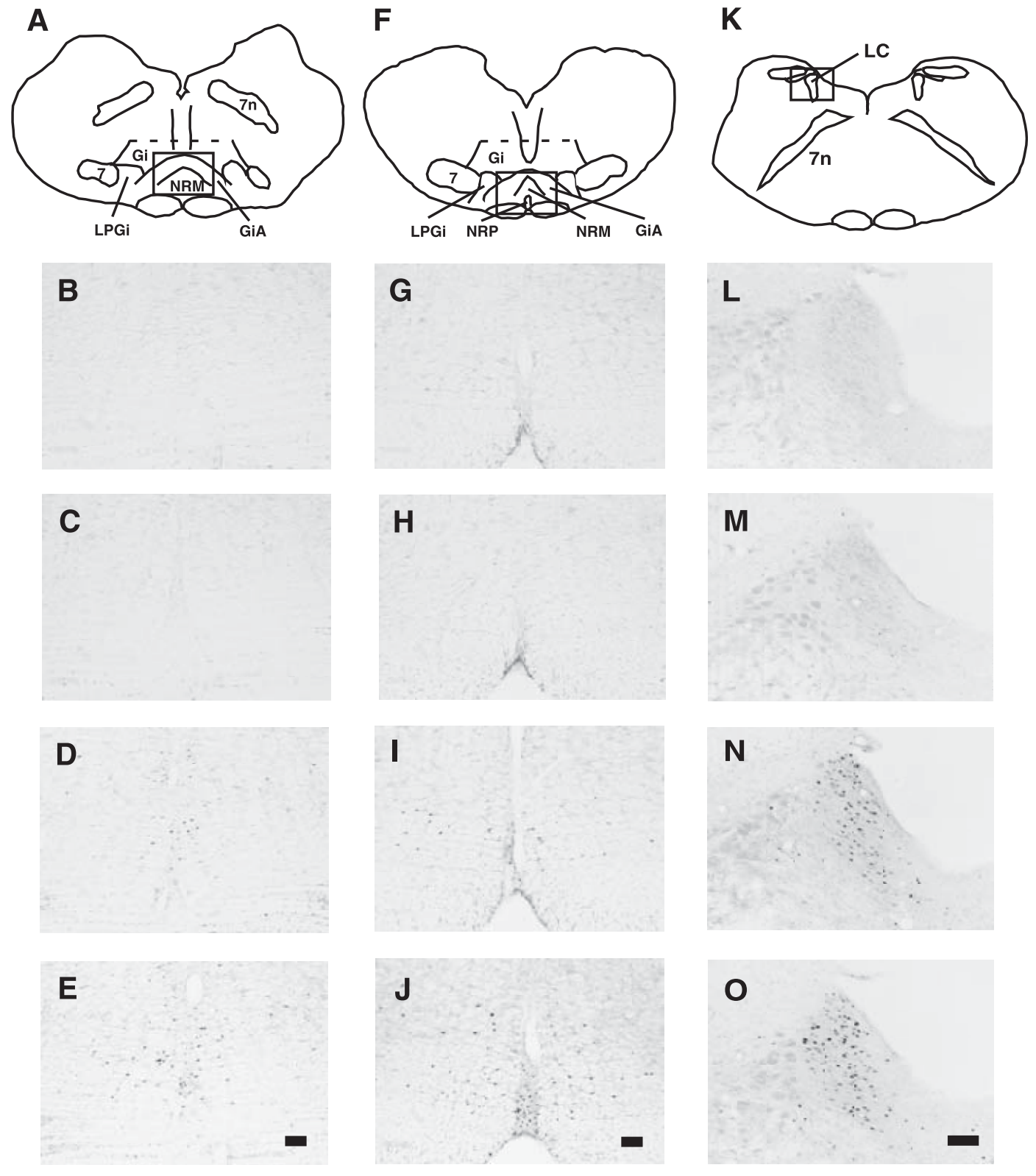

Fig. 2. Schematic illustrations of the rostral RVM (A; bregma $-10.30 \mathrm{~mm})$, caudal RVM (F; bregma $-11.00 \mathrm{~mm})$ and LC (K; bregma $-10.04 \mathrm{~mm}$ ). Photomicrographs showing Fos-LI neurons in the rostral RVM (B, C, D and E corresponding to the boxed area in A), caudal RVM (G, H, I and J corresponding to the boxed area in F) and LC ( L, M, N and O corresponding to the boxed area in $\mathrm{K})$. Histological findings in the TMJ-saline group (B, G and L), TMJ-CFA group (C, H and M), TMJsaline + Masseter-formalin group (D, I and N) and TMJ-CFA + Masseter-formalin group (E, J and O) (7: facial nucleus, 7n: facial nerve, Gi: nucleus reticularis gigantocellularis, GiA: nucleus reticularis gigantocellularis pars alpha, LPGi: nucleus lateralis paragigantocellularis, NRM: nucleus raphe magnus, NRP: nucleus raphe pallidus, LC: locus coeruleus; Scale bars $=100 \mu \mathrm{m})$ indicate the presence of few Fos-LI neurons in the RVM at day 10 after saline $(\mathrm{B}, \mathrm{G}$ and L) or CFA $(\mathrm{C}, \mathrm{H}$ and $\mathrm{M}$ ) injection of the TMJ. However, formalin injection of masseter muscle induces a significant increase in Fos-LI neurons, in particular in the NRM, in the rats with TMJ inflammation (E and J) compared to the rats without TMJ inflammation (D and I).

D and I; Fig. 2: E and J). More Fos-LI neurons were observed in the NRM of the TMJ-CFA + Masseterformalin group (Fig. 2: E and J) than in the TMJsaline + Masseter-formalin group (Fig. 2: D and I). On the other hand, there was a marked increase in Fos-LI neurons in the LC of the groups without and with chronic TMJ inflammation after formalin injection of masseter muscle (Fig. 2: $\mathrm{N}$ and $\mathrm{O}$ ). However, no significant difference in numbers of FosLI neurons in the LC was observed between the TMJ-CFA + Masseter-formalin group and the TMJsaline + Masseter-formalin group. 
A. NRM

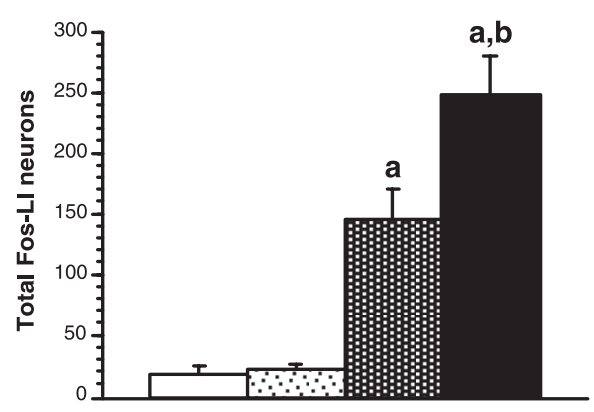

C. Gi

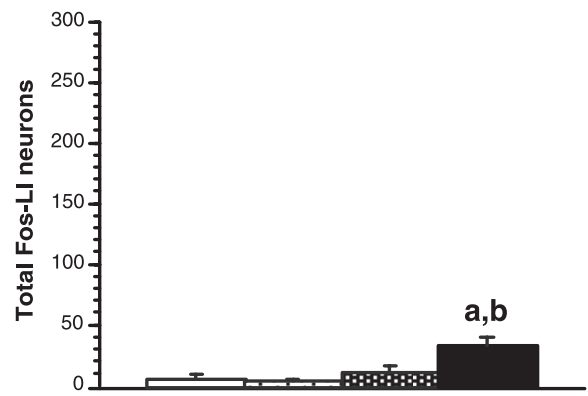

E. LC

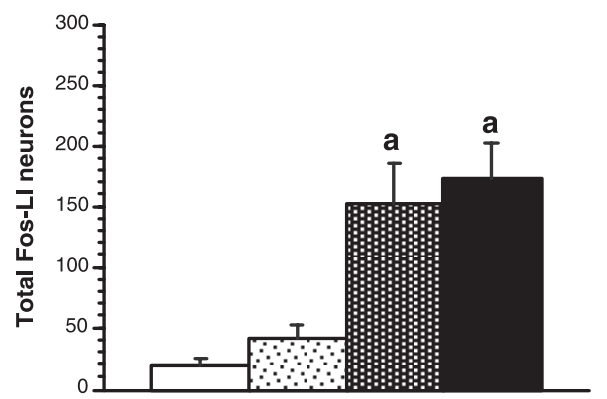

B. GiA

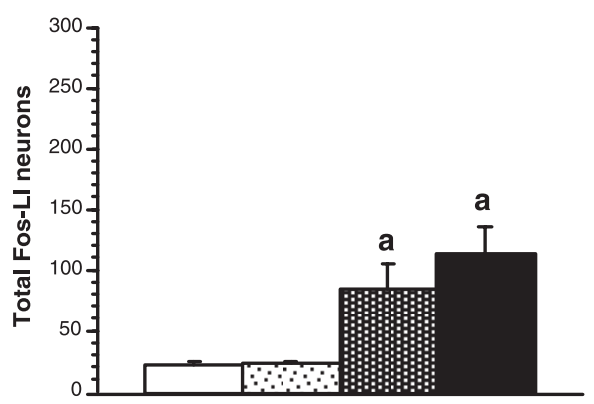

D. NRP

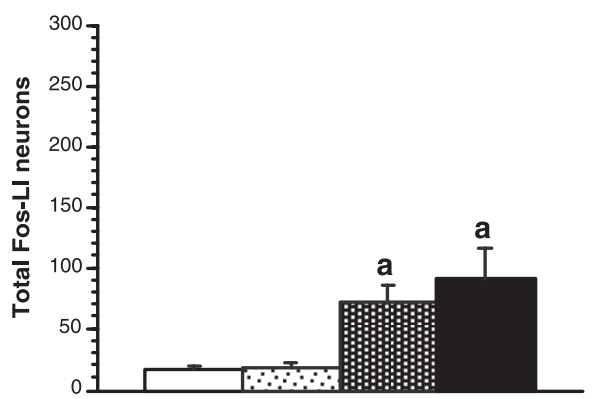

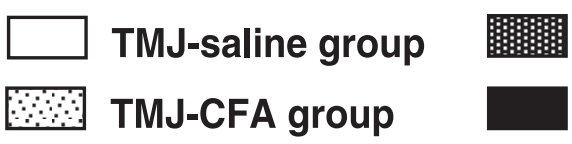

TMJ-saline + Masseter-formalin group TMJ-CFA + Masseter-formalin group

Fig. 3. Effect of chronic TMJ inflammation on Fos expression in the RVM and LC induced by formalin injection of masseter muscle (A: NRM, B: GiA, C: Gi, D: NRP, E: LC; values shown are means \pm S.E.M.). The letter "a" indicates a significant difference between the TMJ-saline group and TMJ-saline + Masseter-formalin group or between the TMJ-CFA group and TMJ-CFA + Masseter-formalin group $(\mathrm{p}<0.05)$. The letter " $b$ " indicates a significant difference between the TMJ-saline + Masseter-formalin group and the TMJ-CFA + Masseter-formalin group $(\mathrm{p}<0.05)$.

We observed that the histological findings in the TMJ-saline + Hindpaw-formalin group and TMJCFA + Hindpaw-formalin group (not shown) were similar to the histological findings in the TMJsaline + Masseter-formalin group (Fig. 2: D, I and $\mathrm{N}$ ) and TMJ-CFA + Masseter-formalin group (Fig. 2: E, J and O), respectively.
2. Analysis of the effect of TMJ-CFA inflammation on Fos-LI in the brainstem

Comparison of the Fos-LI counts in brainstem nuclei revealed that CFA or saline injection of the TMJ induced similar Fos-LI in the TMJ-CFA group (Figs. 3A-E: dotted bars; Table 1) and TMJ-saline group (Fig. 3: A-E: open bars; Table 1). By contrast, 
Table 1. Effect of persistent TMJ inflammation on induction of Fos expression in nuclei by formalin injection of the masseter muscle

\begin{tabular}{lcccc}
\hline & TMJ-saline group & TMJ-CFA group & TMJ-saline + Masseter-formalin group & TMJ-CFA + Masseter-formalin group \\
\hline NRM & $17.5 \pm 7.1$ & $22.0 \pm 3.8$ & $145.8 \pm 25.1^{\mathrm{a}}$ & $248.2 \pm 31.8^{\mathrm{ab}}$ \\
GiA & $22.0 \pm 3.0$ & $22.5 \pm 1.6$ & $84.0 \pm 21.0^{\mathrm{a}}$ & $113.0 \pm 22.9^{\mathrm{a}}$ \\
Gi & $4.8 \pm 3.8$ & $3.5 \pm 1.4$ & $11.0 \pm 4.7$ & $32.4 \pm 8.0^{\mathrm{ab}}$ \\
NRP & $16.8 \pm 1.9$ & $17.0 \pm 4.1$ & $71.8 \pm 14.0^{\mathrm{a}}$ & $90.8 \pm 24.8^{\mathrm{a}}$ \\
LC & $19.3 \pm 5.2$ & $41.5 \pm 10.7$ & $152.8 \pm 33.2^{\mathrm{a}}$ & $173.2 \pm 28.7^{\mathrm{a}}$ \\
\hline
\end{tabular}

Total numbers of Fos-LI neurons in the TMJ-saline group, TMJ-CFA group, TMJ-saline + Masseter-formalin group and TMJCFA + Masseter-formalin group are expressed as means \pm S.E.M. The letter "a" indicates a significant difference between the TMJ-saline + Masseter-formalin group and TMJ-saline group or between the TMJ-CFA + Masseter-formalin group and TMJ-CFA group $(\mathrm{p}<0.05)$. The letter " $\mathrm{b}$ " indicates a significant difference between the TMJ-CFA + Masseter-formalin group and TMJsaline + Masseter-formalin group $(\mathrm{p}<0.05)$.

an increase in Fos-LI counts of $733.1 \%$ in the NRM, $281.8 \%$ in $\mathrm{GiA}, 327.4 \%$ in NRP and $691.7 \%$ in the LC was observed in the TMJ-saline + Masseterformalin group compared to the TMJ-saline group (Table 1). However, no significant differences in total number of Fos-LI neurons in the Gi were found between the TMJ-saline + Masseter-formalin group (Fig. 3C: meshed bar; Table 1) and TMJsaline group (Fig. 3C: open bar; Table 1). Comparison of the Fos-LI neuron counts revealed significantly more Fos-LI neurons in the NRM of the TMJ-CFA + Masseter-formalin group than that of the TMJ-saline + Masseter-formalin group (Fig. 3A; Table 1: $248.2 \pm 31.8$ vs. $145.8 \pm 25.1$ ), and the Gi contained significantly more Fos-LI neurons in the TMJ-CFA + Masseter-formalin group than in the TMJ-saline + Masseter-formalin group (Fig. $3 \mathrm{C}$; Table 1: $32.4 \pm 8.0$ vs. $11.0 \pm 4.7$ ). The results indicated that persistent TMJ inflammation induced an increase in Fos-LI neurons in the NRM and Gi, $70.2 \%$ and $194.5 \%$, respectively, in rats exposed to second noxious stimulus of the masseter muscle (Fig. 3: A and C; closed bars vs. meshed bars; Table $1)$.

There were $874.3 \%, 369.5 \%, 340.5 \%$ and $550.3 \%$ increase in Fos-LI counts in the NRM, GiA, NRP and the LC, respectively, of the TMJsaline + Hindpaw-formalin group than that of the TMJ-saline control group (Fig. 4: A, B, D and E; meshed bars vs. open bars; Table 2). No significant difference in Fos-LI counts in the Gi was found between the TMJ-saline + Hindpaw-formalin group and TMJ-saline group (Fig. 4C; meshed bar vs. open bar; Table 2). On the other hand, comparison of the Fos-LI neuron counts revealed a significant difference in Fos-LI neurons in the NRM between the TMJ-CFA + Hindpaw-formalin group $(262.3 \pm 43.0)$ and TMJ-saline + Hindpaw-formalin groups $(170.5 \pm 25.2)$. There was a $53.8 \%$ increase in Fos-LI neurons in the NRM of the rats with persistent TMJ inflammation and exposed to a sec- ond noxious stimulus of the hindpaw, but no significant increase was found in the Fos-LI neurons in the Gi (Fig. 4: A and C; closed bars vs. meshed bars; Table 2).

\section{Discussion}

Studies on inflammation have demonstrated that deep craniofacial tissue inflammation caused neuronal plastic changes in both the PNS and $\mathrm{CNS}^{14,15,35-37)}$. Peripheral and central sensitization increases the responsiveness and reduces the response threshold of peripheral and central sensory neurons, and contribute to inflammatory hyperalgesia and allodynia. Since the TMJ is innervated by branches of the masseteric nerve, which originates from the mandibular division of the trigeminal nerve, persistent TMJ inflammation causes peripheral sensitization of nerve fibres distributed to the masseter muscle ${ }^{12,13,38)}$. A recent study reported that TMJ inflammation increases nocifensive behaviours induced by formalin injection of the hindpaw of rats, and suggested that inflammation induces central sensitization of supraspinal structures $^{18)}$. Consistent with the results of the behaviour studies, the present study found that persistent TMJ inflammation increased the number of Fos-LI neurons induced by subsequent formalin injection of the masseter muscle or the hindpaw, especially in the NRM. The results of this study suggested that chronic TMJ inflammation gave rise to central sensitization in the descending pain modulatory system as what have been described in some previous clinical studies ${ }^{4,6,7)}$.

Although some studies have demonstrated that Fos expression in the dorsal horn induced by noxious stimulus was modulated by another stimulus previously applied to a spatially remote body areas, how the supraspinal structures being affected was not mentioned ${ }^{17,39-41)}$. However, re-application of 


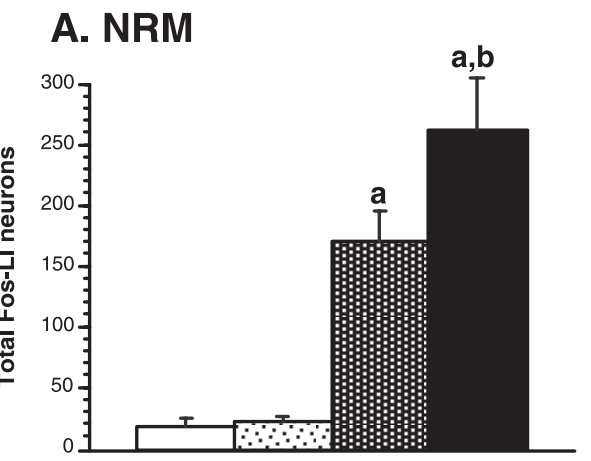

\section{B. GiA}

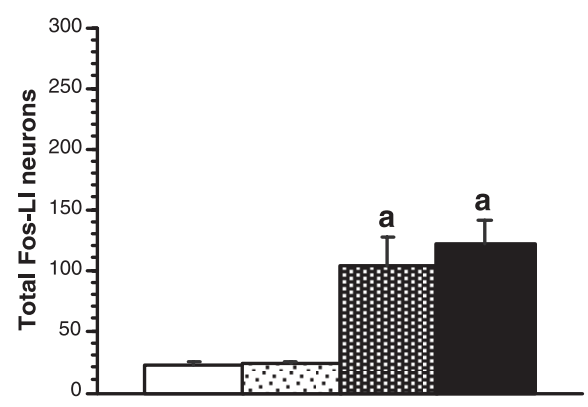

\section{Gi}
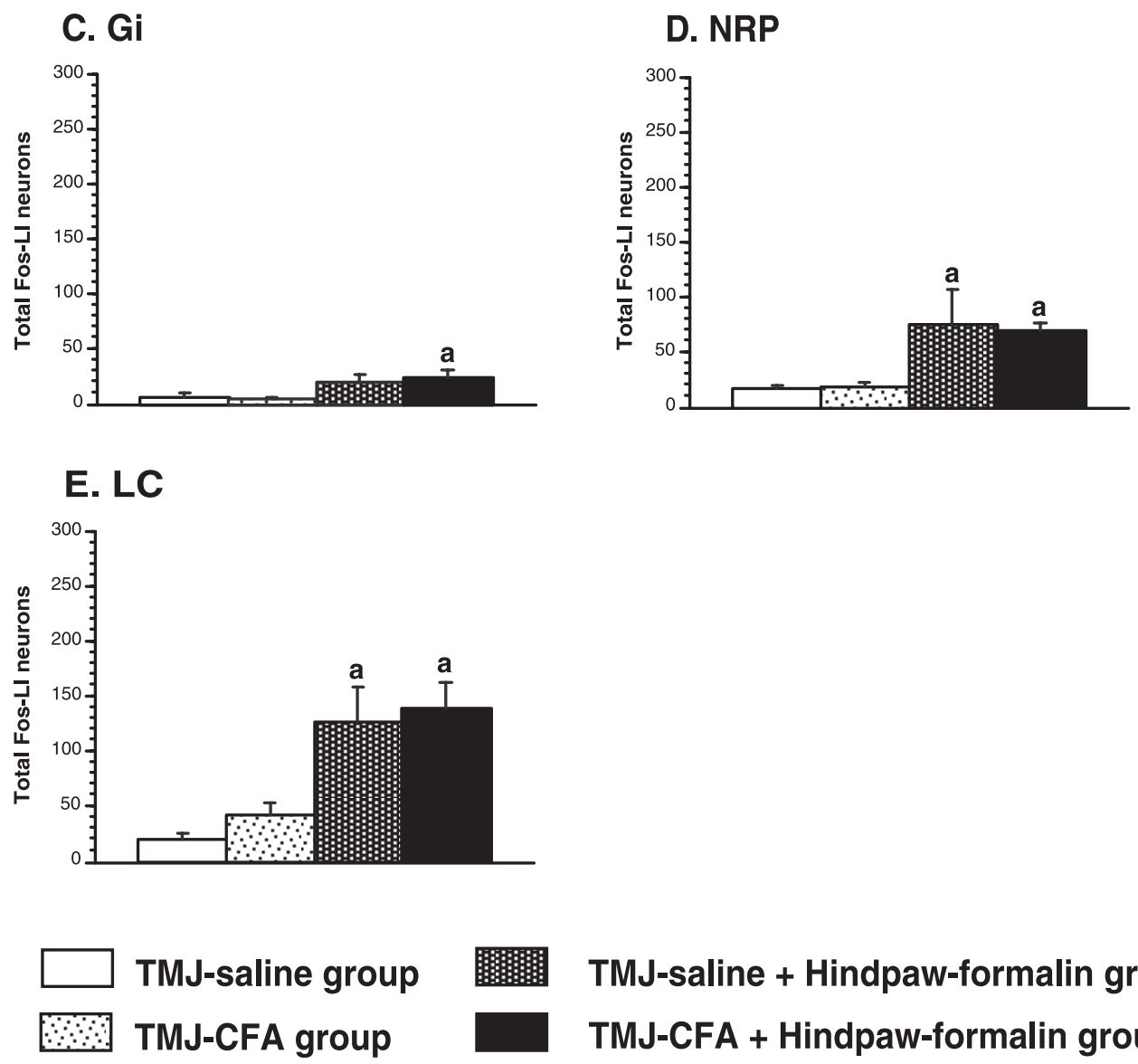

TMJ-saline + Hindpaw-formalin group TMJ-CFA + Hindpaw-formalin group

Fig. 4. Effect of persistent TMJ inflammation on Fos expression in the RVM and LC induced by formalin injection of the hindpaw (A: NRM, B: GiA, C: Gi, D: NRP, E: LC; values shown are means \pm S.E.M.). The letter "a" indicates a significant difference between the TMJ-saline group and the TMJ-saline + Hindpaw-formalin group or between the TMJ-CFA group and the TMJ-CFA + Hindpaw-formalin group $(p<0.05)$. The letter " $b$ " indicates a significant difference between the TMJsaline + Hindpaw-formalin group and the TMJ-CFA + Hindpaw-formalin group $(\mathrm{p}<0.05)$.

a same stimulus 2 weeks after inescapable footshock induced a significant increase in Fos expression in the agranular insular cortex, frontal cortex, basolateral amygdala, CA1 area of the hippocampus, paraventricular hypothalamic nucleus and LC, which are supraspinal structures involved in nociceptive transmission, fear/anxiety, neuroendocrine and autonomic responses ${ }^{42)}$.
The neurons in the RVM were classified into three types in a tail flick reflex (TFL) study: on-cells, which discharge just prior to the TFL, off-cells, which shut off just prior to TFL, and neutralcells, which do not show consistent changes in activity when TFL occurs. On-cell activity facilitates nocifensive behaviours, whereas off-cell activity inhibits them ${ }^{43}$. On the other hand, a 
Table 2. Effect of persistent TMJ inflammation on induction of Fos expression in nuclei by formalin injection of the hindpaw

\begin{tabular}{|c|c|c|c|c|}
\hline & TMJ-saline group & TMJ-CFA group & TMJ-saline + Hindpaw-formalin group & TMJ-CFA + Hindpaw-formalin group \\
\hline NRM & $17.5 \pm 7.1$ & $22.0 \pm 3.8$ & $170.5 \pm 25.2^{\mathrm{a}}$ & $262.3 \pm 43.0^{\mathrm{ab}}$ \\
\hline GiA & $22.0 \pm 3.0$ & $22.5 \pm 1.6$ & $103.3 \pm 23.3^{\mathrm{a}}$ & $121.3 \pm 19.3^{\mathrm{a}}$ \\
\hline $\mathrm{Gi}$ & $4.8 \pm 3.8$ & $3.5 \pm 1.4$ & $19.5 \pm 6.6$ & $23.5 \pm 6.1^{\mathrm{a}}$ \\
\hline NRP & $16.8 \pm 1.9$ & $17.0 \pm 4.1$ & $74.0 \pm 32.4^{\mathrm{a}}$ & $69.3 \pm 6.2^{\mathrm{a}}$ \\
\hline $\mathrm{LC}$ & $19.3 \pm 5.2$ & $41.5 \pm 10.7$ & $125.5 \pm 32.9^{\mathrm{a}}$ & $139.0 \pm 23.0^{\mathrm{a}}$ \\
\hline
\end{tabular}

Total numbers of Fos-LI neurons in the TMJ-saline group, TMJ-CFA group, TMJ-saline + Hindpaw-formalin group and TMJCFA + Hindpaw-formalin group are expressed as means \pm S.E.M. The letter "a" indicates a significant difference between the TMJ-saline + Hindpaw-formalin group and TMJ-saline group or between the TMJ-CFA + Hindpaw-formalin group and TMJCFA group $(\mathrm{p}<0.05)$. The letter " $\mathrm{b}$ " indicates a significant difference between the TMJ-CFA + Hindpaw-formalin group and TMJsaline + Hindpaw-formalin group $(\mathrm{p}<0.05)$.

previous study that related the responses with pawwithdrawal-behaviour test after CFA-induced inflammation identified a significant increase (in percentage) in on-like and off-like cells but a decrease (in percentage) in neutral-like cells in the RVM neurons ${ }^{44)}$. Some studies have reported that formalin injection of the hindpaw and face of rats induces Fos expression in the RVM and $\mathrm{LC}^{27,28)}$. A study of CNS neuronal activity based on glucose utilization showed an increase in neuronal activity in the NRM, GiA, and LC in rats during CFAinduced monoarthritis ${ }^{45}$. A recent study revealed that peripheral inflammation induces activation of extracellular signal-regulated kinases (ERKs), serine/threonine protein kinases play important roles in synaptic plasticity, and suggested that inflammation affects RVM neuronal activity ${ }^{46)}$. The results of the present study suggested that formalin injection of the masseter muscle or hindpaw increased the Fos expression in the NRM, in which increased neuronal reactivity may have been maintained by persistent TMJ inflammation.

It has been reported that Fos expression in NRM serotonergic neurons seems to be implicated in behavioural hyperalgesia, since 5HT released from the descending neurons in the NRM facilitates spinal nociception by activating 5HT3 receptors ${ }^{29,47}$. The results of the present study showed that a second noxious stimulus of the rat masseter or hindpaw differentially induced increase in Fos-LI expression in the RVM nuclei and indicated that Fos-LI expression, particularly in the NRM and Gi, was significantly enhanced by chronic TMJ inflammation. We therefore postulate that increased Fos expression in the NRM is involved in induction and maintenance of the hyperalgesia by spinal 5HT3 receptors. In addition, a previous study on formalin injection of the rat hindpaw demonstrated that 5HT facilitated aversive responses via 5HT3 receptors ${ }^{48)}$.

There is a large group of GiA neurons that in- nervates and modulates NRM activity ${ }^{49)}$. The GiA contains on-cells that are specifically activated by peripheral noxious stimuli and triggered to release GABA onto off-cells to eliminate the tonic inhibition of dorsal horn neurons ${ }^{50}$. There has another study demonstrated that microinjection of cholecystokinin (CCK-8) into the $\mathrm{Gi}$ of naïve rats caused allodynia and hyperalgesia ${ }^{51)}$. By contrast, a chemical lesion of the Gi has been found to lead to attenuation of hyperalgesia and reduce inflammation-evoked spinal Fos expression ${ }^{20)}$. Furthermore, the Gi was assumed to be mainly involved in descending facilitation, because a Gi lesion restored a decreased descending inhibition of inflammation ${ }^{52)}$. The results of the present study also showed an increased masseter-formalininduced Fos-LI in the Gi of TMJ-inflamed rats, and we speculated that it was related to hyperalgesia via descending facilitation of inflammation. However, most of the RVM nuclei seemed to exert a biphasic modulatory effect of nociception, and the increase in formalin-evoked Fos-LI may be a secondary response that counteracts the behavioural hyper$\operatorname{algesia}^{22)}$.

The LC, and A5- and A7-group supraspinal structures contain major noradrenergic neurons that project to the dorsal horn. Electrical stimulation of the structures inhibits dorsal horn nociceptive neurons ${ }^{43)}$. A previous study that used the formalin test reported that long-term removal of spinal noradrenergic afferent axons had no significant effect on nocifensive behaviours ${ }^{53)}$. On the other hand, another study found that stressful stimuli increased Fos-LI neurons in the NRP and LC. The same study demonstrated that NRP neurons project to the intermediolateral cell column and eventually activate the adrenal medulla, whereas LC neurons project to the paraventricular hypothalamic nucleus and eventually activate the adrenal cortex ${ }^{27)}$. We speculated that formalin-evoked Fos-LI, in particular in the NRP and LC, of the 
TMJ-inflamed and non-inflamed rats, might be closely related to the secondary autonomic response counteracting hyperalgesia.

In summary, based on the results of the present study it was concluded that persistent TMJ inflammation increases Fos-LI in the NRM induced by formalin injection of either the ipsilateral trigeminal system (masseter muscle) or a spatially remote body area (hindpaw). The results suggest that chronic TMJ inflammation increases neuronal activity, in particular in the NRM, after exposed to a second noxious stimulus by neuronal plastic change of the descending pain modulatory system.

\section{Acknowledgments}

We would like to express our gratitude to Dr. Keiichiro Okamoto, Department of Physiology, Wakayama Medical University, for his valuable assistance and helpful suggestions in this research.

The Laboratory Animal and Morphological Research Facilities of the Institute of Dental Research, Osaka Dental University, were used to perform this study.

\section{References}

1) Dworkin SF and LeResche L. Research diagnostic criteria for temporomandibular disorders: review, criteria, examinations and specifications, critique. J Craniomandib Disord 1992; 6:301-355.

2) Maixner W, Fillingim R, Booker D and Sigurdsson A. Sensitivity of patients with painful temporomandibular disorders to experimentally evoked pain. Pain 1995; 63:341351.

3) Maixner W, Fillingim R, Sigurdsson A, Kincaid S and Silva S. Sensitivity of patients with temporomandibular disorders to experimentally evoked pain: evidence for altered temporal summation of pain. Pain 1998; 76:71-81.

4) Sarlani $E$ and Greenspan JD. Evidence for generalized hyperalgesia in temporomandibular disorders patients. Pain 2003; 102:221-226.

5) Reid KI, Gracely RH and Dubner RA. The influence of time, facial side, and location on pain-pressure thresholds in chronic myogenous temporomandibular disorder. J Orofac Pain 1994; 8:258-265.

6) Bragdon EE, Light KC, Costello NL, Sigurdsson A, Bunting $\mathrm{S}$, Bhalang $\mathrm{K}$ and Maixner $\mathrm{W}$. Group differences in pain modulation: pain-free women compared to pain-free men and to women with TMD. Pain 2002; 96:227-237.

7) Sarlani E, Grace E, Reynolds M and Greenspan J. Evidence for up-regulated central nociceptive processing in patients with masticatory myofascial pain. J Orofac Pain 2004; 18:41-55.

8) Denucci DJ, Dionne RA and Dubner R. Identifying a neurobiologic basis for drug therapy in TMDs. J Am Dent Assoc 1996; 127:581-593.

9) Lobbezzo F, Drangsholt M, Peck C, Sato H, Kopp S and Svensson P. Topical review: New insights into the pathol- ogy and diagnosis of disorders of the temporomandibular joint. J Orofac Pain 2004; 18:181-191.

10) Ren K. An improved method for assessing mechanical allodynia in the rat. Physiol Behav 1999; 67:711-716.

11) Imbe H, Iwata $K$, Zhou QQ, Zou S, Dubner R and Ren $K$. Orofacial deep and cutaneous tissue inflammation and trigeminal neuronal activation. Cell Tissues Organs 2001; 169:238-247.

12) Okamoto $K$, Imbe $H$, Tashiro $A$, Kumabe $S$ and Senba E. Blockade of peripheral 5HT3 receptor attenuates the formalin-induced nocifensive behavior in persistent temporomandibular joint inflammation of rat. Neurosci Lett 2004; 367:259-263.

13) Okamoto $\mathrm{K}$, Imbe $\mathrm{H}$, Tashiro A, Kimura A, Donishi $T$, Yamai Y and Senba E. The role of peripheral 5HT2A and 5HT1A receptors on the orofacial formalin test in rats with persistent temporomandibular joint inflammation. Neuroscience 2005; 130:465-474.

14) Iwata $K$, Tashiro A, Tsuboi $Y$, Imai $T$, Sumino R, Morimoto $\mathrm{T}$, Dubner R and Ren K. Medullary dorsal horn neuronal activity in rats with persistent temporomandibular joint and perioral inflammation. J. Neurophysiol 1999; 82:1244-1253.

15) Zhou Q, Imbe H, Dubner R and Ren K. Persistent Fos protein expression after orofacial deep or cutaneous tissue inflammation in rats: implications for persistent orofacial pain. J Comp Neurol 1999; 412:276-291.

16) Imbe $H$ and Ren K. Orofacial deep and cutaneous tissue inflammation differentially upregulates preprodynorphin mRNA in the trigeminal and paratrigeminal nuclei of the rat. Brain Res Mol Brain Res 1999; 67:87-97.

17) Bereiter DA, Okamoto K and Bereiter DF. Effect of persistent monoarthritis of the temporomandibular joint region on acute mustard oil-induced excitation of trigeminal subnucleus caudalis neurons in male and female rats. Pain 2005; 117:58-67.

18) Okamoto K, Kimura A, Donishi $\mathrm{T}$, Imbe H, Goda K, Kawanishi K, Tamai Y and Senba E. Persistent monoarthritis of the temporomandibular joint region enhances nocifensive behavior and lumbar spinal Fos expression after noxious stimulation to the hindpaw in rats. Exp Brain Res 2006; 170:358-367.

19) Basbaum AI and Fields HL. Endogenous pain control systems: brainstem spinal pathways and endorphin circuitry. Annu Rev Neurosci 1984; 7:309-338.

20) Wei F, Dubner R and Ren K. Nucleus reticularis gigantocellularis and nucleus raphe magnus in the brain stem exert opposite effects on behavioral hyperalgesia and spinal Fos protein expression after peripheral inflammation. Pain 1999; 80:127-141.

21) Ren K and Dubner R. Descending modulation in persistent pain: an update. Pain 2002; 100:1-6.

22) Zhuo $M$ and Gebhart GF. Biphasic modulation of spinal nociceptive transmission from the medullary raphe nuclei in the rat. J Neurophysiol 1997; 78:746-758.

23) Burgess SE, Gardell LR, Ossipov MH, Malan TP Jr, Vanderah TW, Lai J and Porreca F. Time-dependent descending facilitation from the rostral ventromedial medulla maintains, but does not initiate, neuropathic pain. J Neurosci 2002; 22:5129-5136.

24) Porreca F, Ossipov MH and Gebhart GF. Chronic pain and medullary descending facilitation. Trends Neurosci 2002; 25:319-325.

25) Bullitt E. Expression of c-fos-like protein as a marker for neuronal activity following noxious stimulation in the rat. J Comp Neurol 1990; 296:517-530. 
26) Morgan JI and Curran T. Stimulus-transcription coupling in the nervous system: involvement of the inducible protooncogenes fos and jun. Annu Rev Neurosci 1991; 14:421451.

27) Senba E, Matsunaga K, Tohyama $M$ and Noguchi $K$. Stress-induced c-fos expression in the rat brain: activation mechanism of sympathetic pathway. Brain Res Bull 1993; 31:329-344.

28) Lang B and Li YQ. Serotoninergic neurons in the brainstem expressing FOS protein after orofacial noxious stimulation: an immunocytochemical double-labeling study. J Hirnforsch 1998; 39:263-268.

29) Suzuki R, Morcuende S, Webber M, Hunt SP and Dickenson AH. Superficial NK1-expressing neurons control spinal excitability through activation of descending pathways. Nat Neurosci 2002; 5:1319-1326.

30) Zimmermann M. Ethical guidelines for investigation of experimental pain in conscious animals. Pain 1983; 16:109110.

31) Hutchins B, Spears R, Hinton RJ and Harper RP. Calcitonin gene related peptide and substance $\mathrm{P}$ immunoreactivity in rat trigeminal ganglia and brainstem following adjuvantinduced inflammation of the temporomandibular joint. Arch Oral Biology 2000; 45:335-345.

32) Okamoto $K$, Kimura A, Donishi $T$, Imbe $H$, Senba $E$ and Tamai Y. Central serotonin 3 receptors play an important role in the modulation of nociceptive neural activity of trigeminal subnucleus caudalis and nocifensive orofacial behavior in rats with persistent temporomandibular joint inflammation. Neuroscience 2005; 135:569-581.

33) Paxinos G and Watson C. The Rat Brain in Stereotaxic Coordinates. Fourth edition, Sydney: Academic Press, 1998.

34) Jones BE, Holmes CJ, Rodriguez-Veiga E and Mainville L. GABA-synthesizing neurons in the medulla: their relationship to serotonin-containing and spinally projecting neurons in the rat. J Comp Neurol 1991; 313:349-367.

35) Hu JW, Sessle BJ, Raboisson P, Dallel R and Woda A. Stimulation of craniofacial muscle afferents induces prolonged facilitatory effects in trigeminal nociceptive brainstem neurones. Pain 1992; 48:53-60.

36) Cairns BE, Sessle BJ and $\mathrm{Hu}$ JW. Characteristics of glutamate-evoked temporomandibular joint afferent activity in the rat. J Neurophysiol 2001; 85:2446-2454.

37) Cairns BE, Gambarota G, Svensson P, Arendt-Nielsen L and Berde CB. Glutamate-induced sensitization of rat masseter muscle fibers. Neuroscience 2002; 109:389-399.

38) Klineberg I. Structure and function of temporomandibular joint innervation. Ann R Coll Surg Engl 1971; 49:268-288.

39) Leah JD, Porter J, de-Pommery J, Menetrey D and WeilFuguzza J. Effect of acute stimulation on Fos expression in spinal neurons in the presence of persisting C-fiber activity. Brain Res 1996; 719:104-111.

40) Yang L, Marsala M, Orendacova J and Yaksh T. Knee joint inflammation attenuates spinal FOS expression after unilateral paw formalin injection in rat. Neurosci Lett 1997; 225:89-92.

41) Nagamatsu $N$, Ikeda $T$, Terayama $R$, Nakamura $T$, Koganemaru $\mathrm{M}$ and Nishimori $\mathrm{T}$. Effects of previous noxious stimulus applied to remote areas on noxious stimulusevoked c-Fos expression in the rat trigeminal nucleus caudalis. Neurosci Res 2001; 39:293-298.

42) Bruijnzeel AW, Stam R, Compaan JC, Croiset G, Akkermans LM, Olivier B and Wiegant VM. Long-term sensitization of Fos-responsivity in the rat central nervous system after a single stressful experience. Brain Res 1999; 819:1522.

43) Fields HL and Basbaum AI. Central nervous system mechanisms of pain modulation. In: Wall PD, Melzack R, eds, Text book of pain. London, Harcourt Publishers, 1999; 309-329.

44) Miki K, Zhou QQ, Guo W, Guan Y, Terayama R, Dubner $\mathrm{R}$ and Ren $\mathrm{K}$. Changes in gene expression and neuronal phenotype in brain stem pain modulatory circuitry after inflammation. J Neurophysiol 2002; 87:750-760.

45) Neto FL, Schadrack J, Ableitner A, Castro-Lopes JM, Bartenstein P, Zieglgansberger W and Tolle TR. Supraspinal metabolic activity changes in the rat during adjuvant monoarthritis. Neuroscience 1999; 94:607-621.

46) Imbe $H$, Okamoto $K$, Okamura $T$, Kumabe $S$, Nakatsuka M, Aikawa F, Iwai-Liao Y and Senba E. Effects of peripheral inflammation on activation of ERK in the rostral ventromedial medulla. Brain Res 2005; 1063:151-158.

47) Suzuki R, Rygh LJ and Dickenson AH. Bad news from the brain: descending 5-HT pathways that control spinal pain processing. Trends Pharmacol Sci 2004; 25:613-617.

48) Oyama T, Ueda M, Kuraishi Y, Akaike A and Satoh M. Dual effect of serotonin on formalin-induced nociception in the rat spinal cord. Neurosci Res 1996; 25:129-135.

49) Beitz AJ. The nuclei of origin of brain stem enkephalin and substance $\mathrm{P}$ projections to the rodent nucleus raphe magnus. Neuroscience 1982; 7:2753-2768.

50) Gilbert $\mathrm{AK}$ and Franklin KB. GABAergic modulation of descending inhibitory systems from the rostral ventromedial medulla (RVM). Dose-response analysis of nociception and neurological deficits. Pain 2001; 90:25-36.

51) Kovelowski CJ, Ossipov MH, Sun H, Lai J and Malan TP. Porreca Supraspinal cholecystokinin may drive tonic descending facilitation mechanisms to maintain neuropathic pain in the rat. Pain 2000; 87:265-273.

52) Terayama R, Dubner R and Ren K. The roles of NMDA receptor activation and nucleus reticularis gigantocellularis in the time-dependent changes in descending inhibition after inflammation. Pain 2002; 97:171-181.

53) Jasmin L, Boudah A and Ohara PT. Long-term effects of decreased noradrenergic central nervous system innervation on pain behavior and opioid antinociception. J Comp Neurol 2003; 460:38-55. 